cas ou com restaurações parciais (inlays/ onlays/ overlays) cerâmicas com pino de fibra e preenchimento de resina composta (Rest Parcial pino). Materiais e métodos: Um ensaio clínico controlado randomizado duplo-cego foi conduzido por apenas 1 operador, dentro dos critérios de elegibilidade, randomização e sigilo de alocação. Os critérios de elegibilidade foram: 1) Molares ou pré-molares com tratamento endodôntico e pelo menos uma parede de $3 \mathrm{~mm}$ de espessura; 2) Presença de dentes antagonistas; 3) Margens gengivais do preparo que permitiram adequada moldagem; e 4) Boa higiene bucal. Foram distribuídos aleatoriamente 20 participantes nos dois grupos $(n=40)$, sendo que apenas 1 dente foi incluído por paciente. As peças foram fresadas em IPS e-max CAD, maquiadas e cimentadas. O tempo foi cronometrado em cada sessão, o desconforto foi mensurado por meio da escala VAS e a satisfação foi medida por meio de escala numérica. Análise de regressão de Poisson e análise de variância de medidas repetidas foram realizadas com nível de significância de 5\%. No grupo Rest Parcial pino, $90 \%$ dos participantes relataram pouco ou nenhum desconforto, enquanto 82,5\% do grupo Endocrown respondeu de maneira semelhante. Não houve associação entre as variáveis explicativas (idade, sexo, dente, tipo de tratamento restaurador, número de superfícies tratadas, presença de lesão endodôntica e tempo operatório) e o desconforto relatado pelos pacientes. O nível de satisfação foi de $100 \%$. O tempo operatório no grupo Endocrown $(129,7 \mathrm{~min} \pm 29,78)$ foi semelhante ao do grupo Rest. parcial Pino $(134,1 \mathrm{~min} \pm 29,64)$. Resultados: No grupo Rest Parcial pino, $90 \%$ dos pacientes relataram pouco ou nenhum desconforto, enquanto $82,5 \%$ do grupo Endocrown respondeu de maneira semelhante. Não houve associação entre as variáveis explicativas (idade, sexo, dente, tipo de tratamento restaurador, número de superfícies tratadas, presença de lesão endodôntica e tempo operatório) e o desconforto relatado pelos pacientes. O nível de satisfação foi de $100 \%$. O tempo operatório no grupo Endocrown (129,7 $\min \pm 29,78)$ foi semelhante ao do grupo Rest. parcial Pino $(134,1 \mathrm{~min} \pm 29,64)$. Conclusões: Endocrowns e Rest Parcial pino possuem alto nível de satisfação, baixo desconforto e tempo operatório similar, contudo exigem menos passos clínicos e menor custo final, portanto, as endocrowns são excelentes alternativas para as Rest Parcial pino.

http://doi.org/10.24873/j.rpemd.2019.12.555

\section{\#094 Efetividade de três produtos de branqueamento dentário: ensaio clínico aleatorizado}

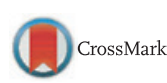

Ruben Pereira*, João Silveira, Susana Dias, Ana Beatriz Cardoso, António Mata, Duarte Marques

Faculdade de Medicina Dentária da Universidade de Lisboa

Objetivos: Avaliar a efetividade clínica de três produtos de branqueamento dentário com diferentes formulações. Materiais e métodos: Foi realizado um ensaio clínico aleatorizado com três grupos paralelos. Foram selecionados trinta pacientes por critérios de inclusão/exclusão, previamente definidos, e foram alocados a um dos três grupos de estudo: $6 \%$ peróxido de hidrogénio em consultório (VivaStyle ${ }^{\circledR}$ Paint On Plus, Ivo-
clarVivadent, Liechtenstein); 16\% peróxido de carbamida em ambulatório com moldeira individual (Opalescence ${ }^{\circledR} \mathrm{PF}$, Ultradent, EUA); 6\% peróxido de hidrogénio em ambulatório com moldeira adaptável (Opalescence ${ }^{\circledR}$ GO, Ultradent, EUA). Todos os voluntários realizaram uma consulta de profilaxia dentária, previamente ao início do tratamento, e a cor dentária dos 12 dentes anteriores de cada voluntário foi registada com um espectrofotómetro (SpectroShade, MHT Optic Research AG, Suiça). Foi realizado um novo registo da cor dentária a meio do tratamento e no final de 12 aplicações (10 minutos cada; 6 por sessão) de VivaStyle ${ }^{\circledR}$ Paint On Plus, 14 aplicações (4 a 6 horas cada) de Opalescence ${ }^{\circledR}$ PF ou 10 aplicações (60 a 90 minutos cada) de Opalescence ${ }^{\circledR} \mathrm{GO}$, segundo instruções do fabricante. Os resultados encontram-se expressos como média e desvio padrão de valores CIE L*a*b* e $\triangle E$. O teste t de Student, one-way ANOVA e Tukey post-hoc foram utilizados conforme apropriado com um nível de significância de $\alpha=0,05$. Resultados: Os valores de $\triangle \mathrm{E}$ para os dentes incisivos e caninos foram de 4,93 $\pm 1,84$ e 7,19 $\pm 1,75$ (VivaStyle ${ }^{\circledR}$ Paint On Plus), 6,84 $\pm 2,10$ e $11,53 \pm 2,84$ (Opalescence ${ }^{\circledR}$ PF) e 3,82 $\pm 2,04$ e 5,97 $\pm 2,35$ (Opalescence $^{\circledR} \mathrm{GO}$ ), respetivamente. Em todos os grupos, os valores CIE L*a*b* obtiveram diferenças estatisticamente significativas entre o início e fim do tratamento $(\mathrm{P}<0,01)$, no entanto na comparação entre grupos apenas estatisticamente significativas $(\mathrm{P}<0,01)$ para o Opalescence ${ }^{\circledR} \mathrm{PF}$ no grupo dos caninos. Conclusões: Todos os produtos demonstraram efeitos clínicos, com maior efetividade no grupo Opalescence ${ }^{\circledR}$ PF. O VivaStyle ${ }^{\circledR}$ Paint On Plus demonstra resultados promissores como técnica a utilizar em consultório, com um menor tempo de aplicação (2x60 minutos) quando comparado com as técnicas de ambulatório. Preconiza-se futuramente, o aumento da amostra em estudo com base na variabilidade obtida, bem como a avaliação da qualidade de vida dos pacientes durante o tratamento. http://doi.org/10.24873/j.rpemd.2019.12.556

\#095 Avaliação da saúde oral e sua relação com o SalivaPrint - Variação com idade e género

Marta André*, Eduardo Esteves, Bruna Correia, Mónica Fernandes, Nuno Rosa

Universidade Católica Portuguesa - Instituto de Ciências da Saúde

Objetivos: A saliva é atualmente utilizada para diagnóstico precoce e monitorização de patologias, tanto orais como sistémicas. No entanto, as estratégias de diagnóstico utilizadas continuam a ser baseadas na identificação de algumas moléculas, muitas vezes inespecíficas de determinada situação. Urge, pois, desenvolver estratégias que permitam avaliar o cenário molecular completo. O SalivaPrint é uma dessas estratégias. Contudo, para que esta abordagem possa ser utilizada no diagnóstico há que padronizar os perfis obtidos e identificar os fatores que afetam este perfil, tais como a idade, o género e a condição oral dos indivíduos. Nesse sentido, este trabalho tem como objetivos: 1. Verificar se há variação do SalivaPrint com a idade e com o género; 2. Verificar se há variação do SalivaPrint com a saúde oral; 3. Verificar se há relação dos parâmetros físico-químicos da saliva com os ín- 
dices de saúde oral. Materiais e métodos: Foi recolhida saliva não estimulada de indivíduos do género feminino e masculino, saudáveis sistémicos e orais dos 7 aos 50 , e um grupo dos 25-50 anos com patologia oral. Foi feita a determinação dos fatores físico-quimos, e dos perfis totais de proteínas por eletroforese capilar em micro-chips. Resultados: Verificou-se que existem alterações do SalivaPrint com a idade e com a saúde oral mas não com o género. A concentração total de proteína não difere, com o género, no entanto difere com a idade. O pH e o fluxo, não diferem, em indivíduos com patologia periodontal nem com a presença de cárie. Uma vez que se observaram diferenças no SalivaPrint de pessoas saudáveis, é importante ter esse facto em conta na comparação com indivíduos doentes para isolar o efeito da doença dos efeitos de outros parâmetros. Conclusões: Este tipo de trabalhos, é essencial para encontrar formas menos invasivas de diagnóstico que tenham em conta toda a variabilidade molecular e fisiológica do indivíduo.

http://doi.org/10.24873/j.rpemd.2019.12.557

\#096 Comportamento celular em Zircónia padronizada por fresa vs laser - estudo in vitro

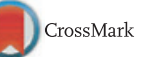

Mariana Brito da Cruz*, Beatriz Ferreira, Joana Faria Marques, António Mata, João Caramês, Filipe Samuel Silva

FMDUL, Departamento de Engenharia Mecânica Universidade do Minho

Objetivos: Comparação do comportamento celular de osteoblastos humanos e fibroblastos gengivais em superfícies texturizadas com sulcos por maquinagem convencional e por laser. Materiais e métodos: Os discos de zircónia foram padronizados por fresas (F) e laser Nd:YAG (L) segundo os grupos de estudo, com diferentes combinações de largura, profundidade e espaçamento (10-150um), sendo o controlo discos sem padrão. Todas as amostras foram tratadas com jateamento e ataque ácido. Os osteoblastos (hFOB 1.19) e os fibroblastos gengivais (HGF) foram cultivados nestas superfícies por 14 dias e a viabilidade celular foi avaliada pela redução da rezasurina. A adesão celular foi confirmada por microscopia eletrónica de varrimento (SEM). O colagénio tipo I, osteopontina (OP) e interleucina 1beta (IL-1b) em hFOB e Interleucina 8 (IL-8) em HGFs foram medidos em tempos pré-determinados por ensaio enzimático de imunoabsorção - ELISA. A atividade da fosfatase alcalina (ALP) foi avaliada em hFOB por técnica enzimática. Os resultados foram apresentados como média \pm desvio padrão. Foram realizadas comparações entre grupos através do teste ANOVA ou teste de Mann-Whitney (post-hoc de Tukey) usando um software de estatística e a significância foi definida como $\mathrm{p}<0,05$. Resultados: A viabilidade dos $\mathrm{hFOB}$ aumentou ao longo do tempo com valores superiores no grupo F em comparação com o grupo L aos 7 e 14 dias $(p<0,05)$. Não se encontraram diferenças entre os grupos na viabilidade dos HGF ( $p>0,05)$. As imagens de SEM revelaram adesão celular após 1 dia de cultura em todas as amostras. Os níveis de colagénio dos hFOB revelaram-se superiores no grupo $F(p<0,05)$, enquanto nos HGF o grupo L foi superior ao grupo $F$ aos 3 dias ( $p<0,05)$, mas não aos 7 dias $(p>0,05)$. Não se encontraram diferenças signi- ficativas na atividade da ALP entre os grupos $(p>0,05)$. A produção de IL-1b foi semelhante em todos os grupos de estudo e não se encontraram diferenças significativas entre o grupo F e L ( $p>0,05)$. Todos os grupos apresentaram um aumento de concentração de OP dos 3 aos 7 dias, superior no grupo $\mathrm{F}$ em comparação com o grupo L ( $\mathrm{p}<0,05)$. A produção da IL-8 por HGFs diminuiu em todos os grupos do $1 .^{\circ}$ ao $3 .^{\circ}$ dia, sem diferenças entre os grupos de estudo em nenhum dos tempos ( $p>0,05)$. Conclusões: As superfícies texturizadas por maquinagem convencional parecem beneficiar a resposta biológica dos osteoblastos em comparação com a fresagem a laser. No entanto, a viabilidade e a diferenciação dos fibroblastos não parecem ser influenciadas pelo tipo de padronização. http://doi.org/10.24873/j.rpemd.2019.12.558

\section{\#097 Incorporação de flúor no esmalte após aplicação de verniz com fluoreto de sódio}

Kateline Dias, Iulian Otel, Micaela Fonseca, Sofia Pessanha, João Silveira*, António Mata

Universidade Europeia, Faculdade de Medicina Dentária da Universidade de Lisboa, Faculdade de Ciência e Tecnologia da Universidade Nova de Lisboa

Objetivos: Este estudo in vitro teve como objectivo avaliar os efeitos da aplicação de um verniz, utilizado no tratamento da sensibilidade dentária, em amostras de esmalte dentário: (1) na incorporação de flúor através da técnica nuclear de PIGE (Particle Induced Gamma-Ray Emission), (2) na desmineralização através de $\mu$-espetroscopia de Raman e (3) na composição elementar por XRF (fluorescência de raios-X). Materiais e métodos: Foram utilizadas 20 amostras de esmalte dentário aleatorizadas em 2 grupos. As amostras de grupo A foram tratadas com um verniz contendo 5\% de flúor (ProFluoride Varnish, VOCO) e uma pasta de dentes não fluoretada. As amostras do grupo B foram sujeitas apenas à escovagem com pasta não fluoretada. As amostras foram analisadas com as técnicas de PIGE, $\mu$-Raman e XRF antes e após a aplicação do verniz. Entre as aplicações, as amostras foram conservadas em saliva artificial. A análise por PIGE foi realizada no acelerador eletrostático Tandem de 3MV. Foi utilizado um feixe de protões a 3,2 $\mathrm{MeV}$ realizando medições em 1 a 2 pontos por amostra. A análise por Raman foi realizada num espetrómetro confocal $\mu$-Raman com fonte de laser díodo de $785 \mathrm{~nm}$ realizando medições em 10 pontos por amostra, de modo a determinar a razão de despolarização da banda de alongamento simétrico do fosfato (960 cm-1). Os resultados de PIGE obtidos encontram-se expressos como ppm (p/p) de flúor no esmalte, a razão de despolarização de $\mu$-Raman encontra-se expressa em unidades arbitrárias e os resultados de XRF encontram-se expressos em $\%$ da composição elementar do esmalte $(\mathrm{p} / \mathrm{p})$. O teste $\mathrm{t}$ de student, one-way ANOVA e Tukey post hoc foram utilizados conforme apropriado e o nível de significância estatística foi estabelecido em $\alpha=0,05$. Resultados: $O$ ratio de despolarização no grupo A, antes e após a aplicação do verniz, diminuiu de forma estatisticamente significativa $(p=0,015)$. No grupo $B$, apesar de a diferença não ser significativa $(p=0,114)$, regista-se uma tendência de aumento de orientação cristalina da hidroxiapatite. 